\title{
A prospective multicenter observational study evaluating the risk of periendoscopic events in patients using anticoagulants: the Osaka GIANT Study
}

\section{(ㄷ)(우우}

Authors

Takuya Inoue1,2, ", Hideki lijima1, ${ }^{\text {, }}$, Takuya Yamada ${ }^{3}$, Yuji Okuyama ${ }^{4}$, Kanae Takahashi ${ }^{5}$, Tsutomu Nishida6,

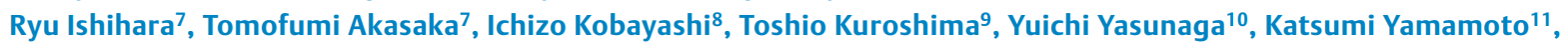
Masanori Nakahara ${ }^{12}$, Yoshinori Doi ${ }^{13}$, Sachiko Nakajima6 ${ }^{6}$, Akira Mukai ${ }^{14}$, Eiji Masuda ${ }^{15}$, Shunsuke Yoshii ${ }^{1}$, Yoshito Hayashi ${ }^{1}$, Hitoshi Minamiguchi ${ }^{4}$, Yasushi Sakata ${ }^{4}$, Kouji Yamamoto ${ }^{5}$, Masahiko Tsujii ${ }^{8}$, Tetsuo Takehara ${ }^{1}$

Institutions

1 Department of Gastroenterology and Hepatology, Osaka University Graduate School of Medicine, Osaka, Japan

2 Department of Gastroenterology, Osaka General Medical Center, Osaka, Japan

3 Department of Gastroenterology, National Hospital Organization Osaka National Hospital, Osaka, Japan

4 Department of Cardiovascular Medicine, Osaka University Graduate School of Medicine, Osaka, Japan

5 Department of Medical Statistics, Osaka City University Graduate School of Medicine, Osaka, Japan

6 Department of Gastroenterology, Toyonaka Municipal Hospital, Toyonaka, Osaka, Japan

7 Department of Gastrointestinal Oncology, Osaka International Cancer Institute, Osaka, Japan

8 Department of Gastroenterology, Higashiosaka City Medical Center, Higashiosaka, Osaka, Japan

9 Department of Gastroenterology, Suita Municipal Hospital, Suita, Osaka, Japan

10 Department of Gastroenterology, Hyogo Prefectural Nishinomiya Hospital, Nishinomiya, Hyogo, Japan

11 Department of Gastroenterology, Japan Community Health Care Organization Osaka Hospital, Osaka, Japan

12 Department of Gastroenterology, Ikeda Municipal Hospital, Ikeda, Osaka, Japan

13 Department of Gastroenterology, Otemae Hospital, Osaka, Japan

14 Department of Gastroenterology, Sumitomo Hospital, Osaka, Japan

15 Department of Gastroenterology, National Hospital Organization Osaka-Minami National Hospital, Osaka, Japan

submitted 28.3.2018

accepted after revision 22.8.2018

\footnotetext{
* These authors contributed equally to the study.
}

Bibliography

DOI https://doi.org/10.1055/a-0754-1997 |

Endoscopy International Open 2019; 07: E104-E114

(c) Georg Thieme Verlag KG Stuttgart · New York

ISSN 2364-3722

Corresponding author

Tetsuo Takehara, MD, PhD, Department of

Gastroenterology and Hepatology, Osaka University

Graduate School of Medicine, 2-2 Yamadaoka, Suita, Osaka

565-0871, Japan

Fax: +81-6-68793629

takehara@gh.med.osaka-u.ac.jp

\section{ABSTRACT}

Background and study aims An increasing number of patients have been using anticoagulants including anti-vitamin $\mathrm{K}$ antagonists (VKAs) and direct oral anticoagulants (DOACs); however, in patients using anticoagulants, limited data are available with regard to the risks of gastrointestinal bleeding and thromboembolic events during the periendoscopic period. We aimed to evaluate the peri-endoscopic bleeding and thrombotic risks in patients administered VKAs or DOACs.

Patients and methods Consecutive patients using anticoagulants who underwent endoscopic biopsy, mucosal resection, or submucosal dissection were prospectively enrolled across 11 hospitals. The primary outcome assessed was difference in incidence of post-procedural gastrointestinal bleeding in patients using VKAs and DOACs. Duration of hospitalization and peri-procedural thromboembolic events were also compared.

Results We enrolled 174 patients using VKAs and 37 using DOACs. In total, 16 patients using VKA were excluded from the analysis because of cancellation of endoscopic procedures and contraindications to the use of DOACs; 128 (81\%) patients using VKAs and $17(46 \%)$ using DOACs received heparin-bridging therapy $(\mathrm{HB})$. The rate of post-procedural gastrointestinal bleeding in DOAC users was similar to that 
in VKA users $(16.2 \%$ vs. $16.4 \%, P=1.000)$. Duration of hospitalization was significantly longer in patients using VKAs than in those using DOACs (median 15 vs. 7 days, $P<0.0001)$. Myocardial infarction occurred during preendoscopic $\mathrm{HB}$ in one patient using VKAs.

Conclusion DOAC administration showed similar postprocedural gastrointestinal bleeding risk to VKA administration in patients undergoing endoscopic procedures, but it shortened the hospital stay.
UMIN-CTR

UMIN000009109

TRIAL REGISTRATION: multi-center, observational and prospective study at umin.ac.jp

\section{Introduction}

Anticoagulants are widely used to treat or prevent thromboembolic events in high risk patients such as those diagnosed with atrial fibrillation, acute coronary syndrome, and deep vein thrombosis [1-5], but are considered strong risk factors for gastrointestinal bleeding with an age- and gender-adjusted hazard ratio of 2.59 [6]. Management of anticoagulant therapy during invasive gastrointestinal endoscopic procedures is a perplexing issue because temporary cessation of antithrombotic drugs may be necessary to reduce the risk of gastrointestinal bleeding, although discontinuation of anticoagulants inversely raises the risk of thrombotic complications [7]. It has been shown that $1.06 \%$ of patients developed cerebral infarction within a month of the endoscopic procedure when use of a vitamin $\mathrm{K}$ antagonist (VKA) such as warfarin was terminated without heparin bridging $(\mathrm{HB})[8]$.

These results have led to a consensus that cessation of anticoagulants should be minimized during endoscopic procedures to prevent life-threatening cerebro-cardiovascular events. Uninterrupted antithrombotic therapy has been recommended for procedures with a low risk for gastrointestinal bleeding, such as endoscopic biopsy $[5,9]$. HB is recommended when high risk endoscopic procedures, such as endoscopic mucosal resection (EMR) and endoscopic submucosal dissection (ESD), are performed [9-11]. However, a study by our group and several other studies have reported that HB in patients using VKAs significantly increased the risk of peri-procedural gastrointestinal bleeding compared to patients who temporarily terminated VKAs without using heparin [12-14]. A recent meta-analysis has shown that VKA-treated patients who received peri-procedural HB demonstrated increased risks of both overall and major bleeding and showed a similar risk of thromboembolic events compared to VKA-treated patients who did not receive HB [15].

Recently, newer anticoagulants including direct thrombin inhibitors (dabigatran) and other direct Factor Xa inhibitors (e.g., rivaroxaban, apixaban, edoxaban) have been used as alternatives to VKA [16-18]. These drugs are collectively termed direct oral anticoagulant drugs (DOACs) or novel oral anticoagulant drugs (NOACs), and have been shown to be effective in preventing cardiogenic cerebral infarction in patients diagnosed with nonvalvular atrial fibrillation $[1,19]$. DOACs show characteristics of a relatively short time to maximal effect and offset of action, and they can be prescribed at fixed doses without the need for close monitoring or dose adjustments $[19,20]$. The rapid onset of anticoagulation and a short half-life of DOACs ensure easier initiation and interruption of anticoagulation than VKAs [21]. To date, most of the available data are retrospective with regard to evaluation of thrombotic and bleeding events during invasive endoscopic procedures in patients using anticoagulants especially for DOACs and even in VKA. Therefore, we conducted a prospective, multicenter observational study, the Osaka Gastrolntestinal Anticoagulant (Osaka GIANT) Study, to evaluate the peri-procedural complications in patients using VKAs and DOACs.

\section{Patients and methods}

\section{Patients and study design}

This is a prospective, multicenter, observational study performed at 1 academic and 10 tertiary care hospitals participating in the Osaka Gut Forum. Patients using anticoagulants who underwent endoscopic procedures (biopsy, EMR, and ESD) were prospectively enrolled in the participating hospitals between September 2012 and December 2015 and these patients were followed up until 30 days after the endoscopic procedures. We obtained informed consent from the patients before endoscopic procedures when invasive endoscopic procedures were scheduled and the patients were registered at the Data Center. When it was uncertain whether endoscopic procedures such as biopsy would be performed, the patients were provisionally pre-registered at the Data Center and were formally registered when the invasive endoscopic procedures were actually performed. All clinical data were entered into case report forms before and after the endoscopic procedure and were submitted to the Data Center. The data analysis was completed in October 2016. This study was registered by the University Hospital Medical Information Network (UMIN) 000009109.

\section{Inclusion and exclusion criteria}

Inclusion criteria for recruitment into the study were: (1) Patients using anticoagulants: warfarin potassium (Warfarin, Eisai, Tokyo, Japan) or DOACs (dabigatran [Pradaxa, Boehringer Ingelheim, Ingelheim, Germany], apixaban [Eliquis, Pfizer Inc., New York, United States], edoxaban [Lixiana, Daiichi Sankyo, Tokyo, Japan], rivaroxaban [Xarelto, Bayer, Leverkusen, Germany]). (2) Patients aged $\geq 20$ years. (3) Patients who required endoscopic biopsy or invasive procedures. 
Exclusion criteria were: (1) Patients with unstable vital signs and a high risk associated with an endoscopic procedure. (2) Patients with apparent gastrointestinal bleeding before the endoscopy. (3) Pregnant women or those breast-feeding, and/ or those with a high possibility of being pregnant. (4) Patients undergoing two or more procedures (biopsy, EMR, and ESD) in the same hospitalization. (5) Patients whose condition was not considered suitable for inclusion in this study. The analysis of post-procedural gastrointestinal bleeding excluded patients using medicines that could possibly interact with DOACs such as itraconazole [22] and those showing an allergy to DOACs. In addition, patients who underwent hemodialysis due to renal dysfunction and those with mechanical heart valve replacement were excluded from the analysis of post-procedural events because those conditions are contraindications to the use of DOACs and would lead to a selection bias to assess VKA.

\section{Assessment of primary and secondary outcomes}

Primary outcome was the prevalence of gastrointestinal bleeding within 30 days after the endoscopic procedures. Secondary outcomes were: (1) Duration of hospitalization. (2) Prevalence of cardiovascular, cerebral, and systemic thromboembolic events. (3) Rate of recurrent gastrointestinal bleeding, which required endoscopic evaluation. (4) Frequency of massive gastrointestinal bleeding. (5) Frequency of fatal gastrointestinal bleeding. (6) Frequency of cerebrovascular bleeding. Gastrointestinal bleeding was defined as the presence of melena or hematemesis, or a drop in hemoglobin level $>2 \mathrm{~g} / \mathrm{dL}$ that was not explained by the presence of other diseases such as oral, nasopharyngeal, and/or anal conditions, and active bleeding or attachment of blood at the lesion of endoscopic treatment or biopsy. Massive bleeding was defined as gastrointestinal bleeding necessitating transfusion of at least 2 units of red cells or symptoms secondary to bleeding in major organs. Fatal bleeding was defined as bleeding into critical sites (intracerebral, subarachnoid, and subdural hemorrhage), bleeding associated with a decrease in hemoglobin level $>5 \mathrm{~g} / \mathrm{dL}$, documented transfusion of at least 9 units of red blood cells, hypotension requiring administration of intravenous catecholamine, and bleeding requiring surgical treatment.

\section{Management of anticoagulants and antiplatelet agents}

In principle, each drug holiday related to oral anticoagulants and antiplatelet drugs before and after performing a therapeutic endoscopy was determined based on the guidelines of the Japan Gastroenterological Endoscopy Society (JGES) [9]. In particular, for endoscopic mucosal biopsy or gastroenterological endoscopic procedures with low bleeding risk, aspirin, nonaspirin antiplatelet agents, or anticoagulants were continued when the patient was on antithrombotic monotherapy. For gastroenterological endoscopic procedures that carried a high risk of bleeding (EMR and ESD), warfarin or dabigatran was recommended to be suspended 3-5 days or 24-48 hours before endoscopy, respectively. There were no recommendations for DOACs other than dabigatran, but most procedures using other DOACs were performed similarly to dabigatran. Requirement of
HB was evaluated by the patient's thromboembolic risk under consultation with specialists, such as cardiologists. Patients who required $\mathrm{HB}$ were instructed to discontinue use of anticoagulants or antiplatelet agents several days before the procedure and continuous intravenous administration of unfractionated heparin (Ajinomoto Pharmaceuticals Co., Ltd., Tokyo, Japan) was initiated after hospitalization. The dose of heparin was adjusted to attain the required activated partial thromboplastin time [9] and administration of heparin sodium was discontinued temporarily at least 3 hours before initiating the endoscopic treatment. After performing endoscopy, heparin sodium was restarted after confirming the absence of melena or development of anemia and its use was discontinued when the international normalized ratio of prothrombin time was observed to be elevated to approximately 1.50 in patients using VKAs. In some cases, the management of anticoagulants did not follow the guidelines, but all of the detailed data on the management of anticoagulants were collected.

\section{Data collection}

The following information was obtained from all patients before the endoscopic procedure: age, sex, height, weight, blood pressure, pulse, results of laboratory tests such as hemoglobin, stroke risk indices (CHADS2 [23], CHA2DS2-VASc [24]), bleeding risk index (HAS-BLED score) [22], name and dose of anticoagulants and antiplatelet drugs used, information regarding cessation of antithrombotic drugs, treatment with HB, history of hypersensitivity to DOACs, comorbidities, details of endoscopic procedures, and details regarding gastrointestinal bleeding. Patients were asked to visit the hospital 30 days after the endoscopic procedures in order to obtain information on subjective symptoms of gastrointestinal bleeding or thromboembolic events as well as information on vital signs and laboratory tests.

\section{Estimation of sample size}

We expected to recruit 450 patients during the study period. The ratio of VKA:DOAC use was 8:1 when generating the study protocol. Therefore, the estimated number of cases in the VKA group was 400 and in the DOAC group was 50 . Our previous study showed that the post-procedural bleeding rate in patients using VKAs who underwent HB was $16 \%$, and that in patients who temporarily terminated VKA use without HB was $3 \%$ [13]. We speculated that the rate of post-procedural bleeding would be similar in patients using DOACs without $\mathrm{HB}$ and in patients using VKAs without HB. The estimated accuracy in determining the bleeding rate was $\pm 3.6 \%$ in the VKA group when the sample size was 400 and $\pm 4.7 \%$ in the DOAC group when the sample size was 50 patients.

\section{Statistical analysis}

Baseline characteristics were expressed as medians (1st quartile, 3 rd quartile) for continuous variables, and proportions for categorical variables. We used Wilcoxon rank-sum test for continuous variables, and Chi-squared test for categorical variables to compare baseline characteristics between the VKA and DOAC groups. Fisher's exact test was used to compare the pro- 


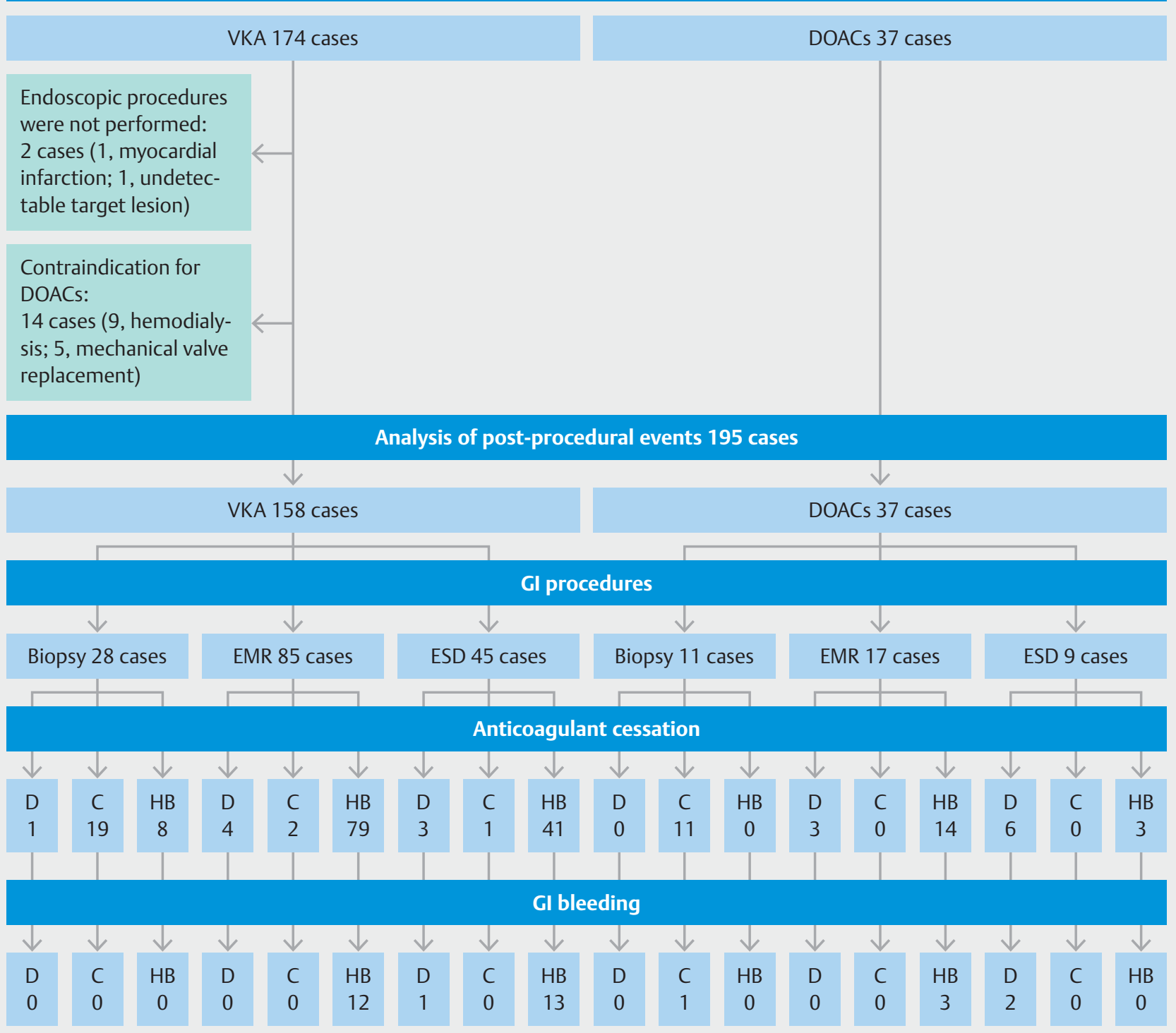

- Fig. 1 Study flow chart and the outcome of post-procedural gastrointestinal bleeding. Patients who received treatment with VKAs and DOACs were included and the numbers of patients with each type of endoscopic procedure, cessation method of anticoagulants, and post-procedural gastrointestinal bleeding are shown. D: discontinue anticoagulants without HB; C: continue anticoagulants without HB; GI: gastrointestinal; HB: heparin bridging.

portion of post-procedural gastrointestinal bleeding between the VKA and DOAC groups. Factors associated with post-procedural gastrointestinal bleeding were assessed using univariable and multivariable logistic regression models with penalized maximum likelihood estimations to correct for possible overfitting of models. These analyses were performed to identify independent risk factors associated with delayed bleeding. To compare the proportion of post-procedural gastrointestinal bleeding among patients with and without peri-procedural heparin bridge therapy, we used Fisher's exact test for each of the VKA and DOAC groups. The difference in the duration of hospital stay between the VKA and DOAC groups was compar- ed using the Wilcoxon rank sum test. The relationship between drugs (VKAs or DOACs) and post-procedural gastrointestinal bleeding was assessed by performing a propensity score analysis with an augmented inverse probability weighted estimator [25] to avoid overfitting that could occur due to inclusion of too many variables into a logistic regression. A $P$ value $<0.05$ was considered to be statistically significant. $R$ version 3.3.2 ( $R$ Foundation for Statistical Computing, Vienna, Austria) was used for multivariate logistic regression models with penalized maximum likelihood estimations and Stata version 14 (College Station, Texas, United States) was used to perform propensity score analysis with an augmented inverse probability weighted 
- Table 1 Patient characteristics.

\begin{tabular}{|c|c|c|c|c|}
\hline & All & VKAs & DOACs & $P$ value \\
\hline Total, n & 195 & 158 & 37 & \\
\hline Age, $y$, median ( $1^{\text {st }}, 3^{\text {rd }}$ quartile $)$ & $74(70,79)$ & $74(70,79)$ & $75(69,78.5)$ & 0.830 \\
\hline Sex, male, n (\%) & $152(77.9)$ & $128(81.0)$ & $24(65.0)$ & 0.033 \\
\hline $\mathrm{CHADS}_{2}$ score, median (range) & $2(0-5)$ & $2(0-5)$ & $2(0-5)$ & 0.356 \\
\hline CHADS $_{2}$-VASc score, median (range) & $4(0-8)$ & $4(0-8)$ & $4(1-7)$ & 0.948 \\
\hline HAS-BLED score, median (range) & $2(0-5)$ & $2(0-5)$ & $2(0-4)$ & 0.810 \\
\hline \multicolumn{5}{|l|}{ Comorbidities } \\
\hline - Atrial fibrillation, n (\%) & $159(81.5)$ & $126(79.7)$ & $33(89.1)$ & 0.183 \\
\hline - Ischemic heart disease, n (\%) & $35(17.9)$ & $29(18.4)$ & $6(16.2)$ & 0.760 \\
\hline - Abnormal liver function test, $\mathrm{n}(\%)$ & $18(9.2)$ & $15(9.5)$ & $3(8.1)$ & 0.793 \\
\hline - Congestive heart failure, n (\%) & $46(23.6)$ & $41(26.0)$ & $5(13.5)$ & 0.581 \\
\hline - Hypertension, n (\%) & $144(73.8)$ & $116(73.4)$ & $28(75.7)$ & 0.778 \\
\hline - Diabetes mellitus, n (\%) & $53(27.1)$ & $41(25.9)$ & $12(32.4)$ & 0.425 \\
\hline - Cerebral infarction/TIA, n (\%) & $45(23.1)$ & $39(24.7)$ & $6(16.2)$ & 0.271 \\
\hline - Renal dysfunction, n (\%) & $63(32.3)$ & $52(32.9)$ & $11(29.7)$ & 0.710 \\
\hline \multicolumn{5}{|l|}{ Anticoagulants } \\
\hline " Warfarin, n (\%) & $158(81.0)$ & $158(100.0)$ & & \\
\hline - DOACs, n (\%) & $37(18.9)$ & & $37(100.0)$ & \\
\hline - Dabigatran, n (\%) & $18(9.2)$ & & $18(48.6)$ & \\
\hline - Rivaroxaban, n (\%) & $9(4.6)$ & & $9(24.3)$ & \\
\hline - Apixaban, n (\%) & $8(4.1)$ & & $8(21.6)$ & \\
\hline - Edoxaban, n (\%) & $2(1.0)$ & & $2(5.4)$ & \\
\hline Antiplatelet drugs, n(\%) & $64(32.8)$ & $52(32.9)$ & $12(27.0)$ & 0.251 \\
\hline - Aspirin, n (\%) & $51(26.1)$ & $44(27.8)$ & $7(18.9)$ & \\
\hline - Clopidogrel, n (\%) & $8(4.1)$ & $5(3.1)$ & $3(8.1)$ & \\
\hline - Others, n (\%) & $11(5.6)$ & $9(5.6)$ & $2(5.4)$ & \\
\hline \multicolumn{5}{|l|}{ Number of antiplatelet drugs } \\
\hline - $0, \mathrm{n}(\%)$ & $131(67.2)$ & $106(67.1)$ & $25(67.6)$ & \\
\hline - $1, \mathrm{n}(\%)$ & $55(28.2)$ & $45(28.5)$ & $10(27.0)$ & \\
\hline - $2, \mathrm{n}(\%)$ & $9(4.6)$ & $7(4.4)$ & $2(5.4)$ & \\
\hline \multicolumn{5}{|l|}{ Region } \\
\hline - Upper gastrointestinal tract, n (\%) & $84(43.0)$ & $65(41.1)$ & $19(51.4)$ & 0.259 \\
\hline \multicolumn{5}{|l|}{ Type of procedure } \\
\hline - EMR,n (\%) & $106(54.3)$ & $89(56.3)$ & $17(45.9)$ & 0.081 \\
\hline - ESD, n (\%) & $47(24.1)$ & $40(25.3)$ & $7(18.9)$ & \\
\hline - Biopsy, n (\%) & $42(21.5)$ & $29(18.4)$ & $13(35.1)$ & \\
\hline
\end{tabular}


- Table 1 (Continuation)

\begin{tabular}{l|l|l|l|}
\hline & All & VKAs & DOACs \\
\hline \begin{tabular}{l} 
Peri-procedural anticoagulants \\
\hline - Termination without heparin bridge, $\mathrm{n}(\%)$
\end{tabular} & $17(8.7)$ & $8(5.0)$ & $9(24.3)$ \\
\hline - Continue, $\mathrm{n}(\%)$ & $33(16.9)$ & $22(13.9)$ & $11(29.7)$ \\
\hline - Heparin bridge, $\mathrm{n}(\%)$ & $145(74.4)$ & $128(81.0)$ & $17(45.9)$ \\
\hline $\begin{array}{l}\text { DOACs, direct oral anticoagulants; EMR, endoscopic mucosal resection; ESD, endoscopic submucosal dissection; TIA, transient ischemic attacks; VKAs, vitamin K } \\
\text { antagonists. }\end{array}$
\end{tabular}

- Table 2 Post-procedural gastrointestinal bleeding in patients treated with VKAs and DOACs.

\begin{tabular}{|c|c|c|c|}
\hline & $\begin{array}{l}\text { VKAs } \\
(n=158)\end{array}$ & $\begin{array}{l}\text { DOACs } \\
(n=37)\end{array}$ & $P$ value* \\
\hline Total post-procedural bleeding, n (\%) & $26(16.4)$ & $6(16.2)$ & 1.000 \\
\hline Massive bleeding, n (\%) & $14(8.8)$ & $3(8.1)$ & 1.000 \\
\hline Fatal bleeding, n (\%) & $2(1.3)$ & $0(0.0)$ & 1.000 \\
\hline
\end{tabular}

estimator. Other statistical analyses were performed using JMP 12.2.0 software (SAS Institute, North Carolina, United States).

\section{Ethical considerations}

This study was performed in accordance with the Declaration of Helsinki and was approved by the Ethics Committees of Osaka University Hospital and participating hospitals. Written informed consent was obtained from each patient before performing the endoscopic procedures.

\section{Results}

\section{Characteristics of patients and endoscopic procedures}

The study was terminated before reaching the expected number of patients. Our study included 211 patients including 174 patients who used VKAs and 37 who used DOACs. Endoscopic treatment was not performed in two patients: one patient suffered a myocardial infarction during the cessation period of VKAs before the endoscopic procedure, and in one patient, the target lesion was not detected on the day of the endoscopic procedure. We also excluded 9 patients who underwent hemodialysis and 5 who underwent mechanical heart valve replacement (the use of DOACs was contraindicated) from the analysis of post-procedural events. Finally, 195 patients (158 VKAs and 37 DOACs) were analyzed to compare post-procedural gastrointestinal bleeding ( $\triangleright$ Fig.1). The characteristics of patients analyzed for post-procedural events are shown in $>$ Table 1 . The VKA group included a greater proportion of men than those in the DOAC group ( $81.0 \%$ vs. $65.0 \%, P=0.033$ ). A significantly larger proportion of patients received $H B$ in the VKA group than in the DOAC group $(81.0 \%$ vs. $45.9 \%, P<0.0001$; - Table 1). There were no statistically significant differences in other background characteristics between the two groups.

\section{Post-procedural gastrointestinal bleeding in patients using vitamin $\mathrm{K}$ antagonists and direct oral anticoagulants}

The total post-procedural gastrointestinal bleeding rates within 30 days of an endoscopic procedure did not differ significantly between the VKA and the DOAC groups (16.4\% vs. $16.2 \%, P=1.000 ; \triangleright$ Table 2$)$. The proportion of patients with an abnormal liver function test was similar between the two groups ( $\triangleright$ Table 1). Four patients had a history of hematological disorders in the VKA group and none in the DOAC group. Both patients with liver dysfunction and hematological disorders did not experience post-procedural gastrointestinal bleeding. The post-procedural gastrointestinal bleeding rates and the proportion of patients who received $\mathrm{HB}$ associated with each type of DOAC use are shown in > Supplementary Table 1. The rates of massive and fatal bleeding did not differ significantly between the two groups ( $>$ Table 2 ). All patients who developed massive and fatal bleeding were successfully treated using endoscopic hemostasis. There was no difference in the duration of bleeding after the endoscopic procedures and the rate of recurrent bleeding observed between patients using VKAs and DOACs ( $\triangleright$ Supplementary Table 2). No patient from either group developed any major extra-gastrointestinal bleeding.

To further confirm the anticoagulant-induced risks of postprocedural gastrointestinal bleeding, we compared the rate of gastrointestinal bleeding in matched patients receiving VKAs and DOACs using propensity score analysis with an augmented 
- Table 3 Characteristics of the patients with and without post procedural gastrointestinal bleeding.

\begin{tabular}{|c|c|c|}
\hline & Without gastrointestinal bleeding $(n=163)$ & With gastrointestinal bleeding $(n=32)$ \\
\hline DOACs, n (\%) & $31(19.0)$ & $6(18.8)$ \\
\hline Age, $y$, median ( $1^{\text {st }}, 3^{\text {rd }}$ quartile) & $75.0(70.0,79.0)$ & $73.0(67.8,77.3)$ \\
\hline Sex, male, n (\%) & $125(76.7)$ & $27(84.4)$ \\
\hline Region, upper gastrointestinal tract, $\mathrm{n}(\%)$ & $68(41.7)$ & $16(50.0)$ \\
\hline Procedure, EMR, n (\%) & $90(55.2)$ & $16(50.0)$ \\
\hline Procedure, ESD, n (\%) & $33(20.2)$ & $14(43.8)$ \\
\hline Procedure, biopsy, n (\%) & $40(24.5)$ & $2(6.2)$ \\
\hline With heparin bridge, $\mathrm{n}(\%)$ & $113(69.3)$ & $26(81.2)$ \\
\hline Atrial fibrillation, $\mathrm{n}(\%)$ & $132(81.0)$ & $27(84.4)$ \\
\hline Ischemic heart disease, $n(\%)$ & $27(16.6)$ & $8(25.0)$ \\
\hline Congestive heart failure, $\mathrm{n}(\%)$ & $38(23.3)$ & $9(28.1)$ \\
\hline Hypertension, n (\%) & $123(75.5)$ & $21(65.6)$ \\
\hline Diabetes mellitus, n (\%) & $47(28.8)$ & $6(18.8)$ \\
\hline Cerebral infarction/TIA, n (\%) & $36(22.1)$ & $9(28.1)$ \\
\hline Abnormal liver function test, $\mathrm{n}(\%)$ & $18(11.0)$ & $0(0.0)$ \\
\hline Renal dysfunction, $\mathrm{n}(\%)$ & $55(33.7)$ & $8(25.0)$ \\
\hline Antiplatelet drugs/NSAID use, $\mathrm{n}(\%)$ & $50(30.7)$ & $11(34.4)$ \\
\hline
\end{tabular}

inverse probability weighted estimator. We used a logit model to predict the use of drugs (VKAs or DOACs) as a function of age, sex, size of the lesion, present or past history of hemorrhagic diathesis, serum creatinine, liver function tests, and type of endoscopic procedure, and we used a logit regression model for post-procedural gastrointestinal bleeding, using HB and endoscopic procedures as explanatory variables. This propensity score analysis showed no statistically significant difference between patients using VKAs and DOACs in terms of the mean treatment effect on post-procedural gastrointestinal bleeding $(P=0.108)$.

\section{Univariable and multivariable analysis showing risk factors associated with post-procedural gastrointestinal bleeding}

We further investigated the role of anticoagulants and other factors associated with post-procedural gastrointestinal bleeding in patients using anticoagulants by dividing patients into groups based on those with and those without post-procedural gastrointestinal bleeding. The characteristics of patients with and without post-procedural gastrointestinal bleeding are shown in > Table 3. Univariable analysis revealed an ESD was a significant risk factor associated with post-procedural gastrointestinal bleeding ( $\triangleright$ Table 4). Multivariable analysis, however, revealed that none of the selected factors were associated with post-procedural gastrointestinal bleeding ( $>$ Table 4).

\section{Risk of post-procedural gastrointestinal bleeding according to use of heparin bridging}

We next compared the post-procedural gastrointestinal bleeding rates between the VKA and DOAC groups among the subgroups of patients who did and did not receive HB ( Supplementary Table 3). There was no significant difference in the gastrointestinal bleeding rate between VKA and DOAC groups. Next, we compared the gastrointestinal bleeding rate between patients in each group separately among those who did and did not receive $\mathrm{HB}$. The post-procedural gastrointestinal bleeding rate was significantly higher in patients who received $\mathrm{HB}$ than in those without HB in the VKA group ( $P=0.049$, $>$ Table 5); however, it did not differ significantly in those using DOACs.

\section{Assessment of peri-procedural thromboembolic events in patients using vitamin $\mathrm{K}$ antagonists versus direct oral anticoagulants}

Among the 211 patients initially registered for the study, one patient from the VKA group suffered a myocardial infarction during the phase of temporary termination of VKAs. No patient reported cerebro-cardiovascular thromboembolic and bleeding events during the study period. Overall, the incidence of cerebro-cardiovascular thromboembolic events was not statistically significantly different between the VKA and DOAC groups $(0.6 \%[1 / 174]$ vs. $0 \%$ [0/37], $P=0.644)$. 
Table4 Univariable and multivariable analysis of the factors associated with post-procedural gastrointestinal bleeding.

\begin{tabular}{|c|c|c|c|c|c|c|c|}
\hline & \multirow[b]{2}{*}{ Category } & \multicolumn{3}{|l|}{ Univariable } & \multicolumn{3}{|c|}{ Multivariable } \\
\hline & & Odds ratio & $95 \% \mathrm{Cl}$ & $P$ value & Odds ratio & $95 \% \mathrm{Cl}$ & $P$ value \\
\hline DOACs & Yes/no & 0.983 & $0.372-2.59$ & 0.972 & 1.666 & $0.512-5.42$ & 0.397 \\
\hline Age & 1 year & 0.724 & $0.464-1.13$ & 0.154 & 0.664 & $0.398-1.11$ & 0.117 \\
\hline Sex & Male/female & 0.609 & $0.219-1.69$ & 0.341 & 2.617 & $0.242-28.27$ & 0.658 \\
\hline Region & $\begin{array}{l}\text { Upper gastrointes- } \\
\text { tinal/lower gastro- } \\
\text { intestinal tract }\end{array}$ & 1.397 & $0.654-2.99$ & 0.388 & 1.766 & $0.507-6.16$ & 0.428 \\
\hline Heparin bridge & Yes/no & 1.917 & $0.743-4.95$ & 0.178 & 1.011 & $0.091-11.26$ & 0.372 \\
\hline Atrial fibrillation & Yes/no & 0.789 & $0.281-2.21$ & 0.652 & 3.133 & $0.870-11.29$ & 0.326 \\
\hline Ischemic heart disease & Yes/no & 1.679 & $0.682-4.13$ & 0.259 & 0.792 & $0.282-2.22$ & 0.729 \\
\hline Congestive heart failure & Yes/no & 1.287 & $0.549-3.02$ & 0.561 & 0.578 & $0.194-1.72$ & 0.274 \\
\hline Hypertension & Yes/no & 1.611 & $0.715-3.63$ & 0.250 & 1.667 & $0.667-4.16$ & 0.603 \\
\hline Diabetes mellitus & Yes/no & 0.570 & $0.220-1.47$ & 0.246 & 0.750 & $0.176-3.20$ & 0.213 \\
\hline Cerebral infarction/TIA & Yes/no & 1.380 & $0.587-3.25$ & 0.460 & 1.274 & $0.512-3.17$ & 0.599 \\
\hline Renal dysfunction & Yes/no & 0.655 & $0.276-1.55$ & 0.336 & 1.276 & $0.514-3.17$ & 0.215 \\
\hline Antiplatelet drugs/NSAID use & Yes/no & 1.184 & $0.531-2.64$ & 0.680 & 2.352 & $0.706-7.83$ & 0.802 \\
\hline Endoscopic procedure & EMR/biopsy & 0.528 & $0.232-1.20$ & 1.000 & 0.548 & $0.212-1.42$ & 0.993 \\
\hline Endoscopic procedure & ESD/biopsy & 3.724 & $1.144-12.12$ & 0.029 & 1.125 & $0.449-2.81$ & 0.081 \\
\hline
\end{tabular}

$\mathrm{CI}$, confidence interval; DOACs, direct oral anticoagulants; EMR, endoscopic mucosal resection; ESD, endoscopic submucosal dissection; NSAID, non-steroidal antiinflammatory drugs; TIA, transient ischemic attacks.

- Table 5 Rate of post-procedural bleeding in patients taking VKAs or DOACs with/without peri-procedural heparin bridge therapy.

\begin{tabular}{|l|l|l|l|}
\hline & Without heparin bridge & With heparin bridge & $\boldsymbol{P}^{*}$ \\
\hline VKAs, $\mathrm{n}(\%)$ & $1 / 29(3.5)$ & $25 / 129(19.4)$ & 0.049 \\
\hline DOACs, $\mathrm{n}(\%)$ & $3 / 20(15.0)$ & $3 / 17(17.7)$ & 1.000 \\
\hline $\begin{array}{l}\text { Cl, confidence interval; DOACs, direct oral anticoagulants; VKAs, vitamin K antagonists. } \\
\text { * Fisher's exact test. }\end{array}$ & \\
\hline
\end{tabular}

- Table 6 Duration of hospital stay in patients taking VKAs or DOACs.

\begin{tabular}{|c|c|c|c|c|c|}
\hline & VKAs & DOACs & Difference between groups & $95 \% \mathrm{Cl}$ & $P$ value* \\
\hline Duration (range) & $15(1-48)$ & $7(227)$ & 8.69 & $5.49-11.90$ & $<0.0001$ \\
\hline
\end{tabular}

\section{Duration of hospital stay for patients using vitamin $\mathrm{K}$ antagonists versus those using direct oral anticoagulants}

The proportion of patients who received HB was significantly lower in the DOAC group than in the VKA group ( $>$ Table 1 ), and the duration of hospitalization was significantly shorter in the DOAC group than in the VKA group (median 7 vs. 15 days, $P<0.0001$; Table 6).

\section{Discussion}

DOACs are increasingly used as substitutes for VKAs because they obviate the need for dose adjustment and lower the risk of life-threatening bleeding and intracranial hemorrhage [26]. However, DOAC users are reportedly at a higher risk for the development of gastrointestinal hemorrhagic events than VKA users [27]. In this prospective, multicenter observational study, the rate of post-procedural gastrointestinal bleeding in pa- 
tients using DOACs was similar to that in those using VKAs. We previously reported that $\mathrm{HB}$ increases the risk of post-procedural gastrointestinal bleeding in patients undergoing gastric ESD and colonic EMR $[12,13]$, and we initially speculated that concomitant use of VKA and heparin may increase the risk of postprocedural gastrointestinal bleeding. We therefore reckoned that DOACs, which can avoid or minimize heparin use, can reduce post-procedural gastrointestinal bleeding. Contrary to expectations, DOAC use showed a similar incidence rate of post-procedural gastrointestinal bleeding to that of VKA use with $\mathrm{HB}$ ( $\triangleright$ Table 5 ). Due to the short pre-intervention termination period and strong antithrombotic effects, the risk of gastrointestinal bleeding associated with DOACs may be similar to that associated with VKAs used with $\mathrm{HB}$. A recent meta-analysis has revealed that DOACs are associated with a higher risk of gastrointestinal bleeding than the standard care using VKAs [27]. Based on these results, it is important for endoscopists to be aware of the risk of post-procedural gastrointestinal bleeding in patients using DOACs even in the absence of $\mathrm{HB}$, which is similar to that in patients using VKA with $\mathrm{HB}$.

The American Society for Gastrointestinal Endoscopy (ASGE) guidelines and JGES guidelines published in 2012 recommend bridge therapy for patients using VKAs before undergoing high risk endoscopic procedures in those who are at high risk for the development of thromboembolic events [5, 9]. However, previous studies by our own group have shown a high risk of gastrointestinal bleeding after $\mathrm{HB}[12,13,28]$. Similar to these retrospective studies, our prospective study confirmed a higher risk of post-procedural gastrointestinal bleeding in patients using VKAs who received $\mathrm{HB}$ than in those who did not receive $\mathrm{HB}$. Moreover, we observed that a patient from the VKA group suffered acute myocardial infarction during the temporary termination phase of anticoagulant therapy, although HB had been performed. The recently reported BRIDGE clinical trial, which included approximately $50 \%$ of patients who underwent minor gastrointestinal procedures (low bleeding risk), revealed that forgoing peri-operative bridging anticoagulation was not inferior to peri-operative HB to prevent arterial thromboembolism and decrease the risk of major bleeding [29]. Recent ASGE guidelines recommend HB in patients using DOACs who are at a high risk for the development of thromboembolic events and that they undergo high risk endoscopic procedures only when a DOAC cannot be restarted within 24 hours after the endoscopic procedure has been performed [5]. European Society of Gastrointestinal Endoscopy (ESGE) guidelines do not recommend $\mathrm{HB}$ in patients using DOACs due to their fast on and off effects [21]. The recently revised Japanese guidelines also allow continuous use of VKA or DOAC use without $\mathrm{HB}$ in high risk procedures [30]. A recent prospective study, which primarily included non-therapeutic endoscopic procedures, showed that short-term interruption of DOACs was safe [31]. Temporary termination of DOACs and forgoing HB can theoretically be allowed because of their short T-max and halflife and can reduce the duration of hospitalization without increasing gastrointestinal bleeding and thromboembolic risks.

Our current study has several limitations: (1) This study was terminated before the recruitment of the expected number of the patients due to the scarcity of patients. Some of the cases with biopsy, which were not planned in advance, may be missed for registration because informed consent needed to be obtained before the endoscopic procedures. In addition, endoscopists may be hesitant to perform biopsy in cases with a low risk of malignancy judged from the endoscopic appearance. Although we could not collect the expected number of patients, our data showed that the bleeding rate in DOACs was much higher than we initially estimated and these data were considered very important in the situation of increasing DOAC users. (2) Because the sample size was small, our subgroup analysis of DOACs was not adequately performed. (3) We used intravenously administered unfractionated heparin because use of low-molecular-weight heparin (LMWH) is limited during hemodialysis and for the treatment of disseminated intravascular coagulation in our country, although use of LMWH is recommended in the ASGE and ESGE guidelines [5, 10]. LMWH can be used in an outpatient setting $[32,33]$ and the benefit of reduced duration of hospitalization may not be applicable to LMWH; however, using DOACs reduce heparin use and simplify the process of anticoagulant administration. (4) Most of the procedures were performed under the guidance of JGES guidelines, but some were performed by the decision of their own institution as some physicians were still concerned about gastrointestinal bleeding after endoscopic procedures with continuation of anticoagulants. Because sufficient evidence was still not available for the use of anticoagulant drugs, especially prospective studies, we conducted a prospective study and confirmed that continuation of anticoagulants is possible in low risk procedures such as biopsy.

In conclusion, peri-endoscopic procedural complications in patients taking DOACs were similar to those in patients taking VKAs; however, use of DOACs is shown to be beneficial because this class of drugs can significantly reduce the duration of hospitalization compared to VKA with HB.

\section{Competing interests}

These authors have the following financial conflicts of interest regarding this manuscript. Hideki lijima, Yasushi Sakata, and Tetsuo Takehara received funding from Daiichi-Sankyo.

References

[1] Yaghi S, Kamel H, Elkind MS. Potential new uses of non-vitamin $\mathrm{K}$ antagonist oral anticoagulants to treat and prevent stroke. Neurology 2015; 85: $1078-1084$

[2] Nagata N, Niikura R, Aoki T et al. Effect of proton-pump inhibitors on the risk of lower gastrointestinal bleeding associated with NSAIDs, aspirin, clopidogrel, and warfarin. J Gastroenterol 2015; 50: 10791086

[3] Fujita M, Shiotani A, Murao T et al. Safety of gastrointestinal endoscopic biopsy in patients taking antithrombotics. Dig Endosc 2015; 27: $25-29$

[4] Sterne JA, Bodalia PN, Bryden PA et al. Oral anticoagulants for primary prevention, treatment and secondary prevention of venous throm- 
boembolic disease, and for prevention of stroke in atrial fibrillation: systematic review, network meta-analysis and cost-effectiveness analysis Health Technol Assess 2017; 21: 1-386

[5] Acosta RD, Abraham NS, Chandrasekhara V et al. The management of antithrombotic agents for patients undergoing Gl endoscopy. Gastrointest Endosc 2016; 83: 3-16

[6] Kaplan RC, Heckbert SR, Koepsell TD et al. Risk factors for hospitalized gastrointestinal bleeding among older persons. Cardiovascular Health Study Investigators. J Am Geriatr Soc 2001; 49: 126-133

[7] Wahl M]. Dental surgery in anticoagulated patients. Arch Intern Med 1998; 158: 1610 - 1616

[8] Blacker D], Wijdicks EF, McClelland RL. Stroke risk in anticoagulated patients with atrial fibrillation undergoing endoscopy. Neurology 2003; 61: $964-968$

[9] Fujimoto K, Fujishiro M, Kato M et al. Guidelines for gastroenterological endoscopy in patients undergoing antithrombotic treatment. Dig Endosc 2014; 26: 1-14

[10] Veitch AM, Baglin TP, Gershlick AH et al. Guidelines for the management of anticoagulant and antiplatelet therapy in patients undergoing endoscopic procedures. Gut 2008; 57: 1322 - 1329

[11] Anderson MA, Ben-Menachem T, Gan SI et al. Management of antithrombotic agents for endoscopic procedures. Gastrointest Endosc 2009; 70: 1060 - 1070

[12] Yoshio T, Nishida T, Kawai N et al. Gastric ESD under heparin replacement at high-risk patients of thromboembolism is technically feasible but has a high risk of delayed bleeding: Osaka University ESD Study Group. Gastroenterol Res Pract 2013; 2013: 365830

[13] Inoue T, Nishida T, Maekawa A et al. Clinical features of post-polypectomy bleeding associated with heparin bridge therapy. Dig Endosc 2014; 26: 243-249

[14] Kubo T, Yamashita K, Onodera K et al. Heparin bridge therapy and post-polypectomy bleeding. World J Gastroenterol 2016; 22: 1000910014

[15] Siegal D, Yudin J, Kaatz S et al. Periprocedural heparin bridging in patients receiving vitamin $\mathrm{K}$ antagonists: systematic review and meta-analysis of bleeding and thromboembolic rates. Circulation 2012; 126: $1630-1639$

[16] Turun S, Banghua L, Yuan Y et al. A systematic review of rivaroxaban versus enoxaparin in the prevention of venous thromboembolism after hip or knee replacement. Thromb Res 2011; 127: 525 - 534

[17] Cao YB, Zhang JD, Shen $\mathrm{H}$ et al. Rivaroxaban versus enoxaparin for thromboprophylaxis after total hip or knee arthroplasty: a meta-analysis of randomized controlled trials. Eur J Clin Pharmacol 2010; 66: 1099-1108

[18] Neumann I, Rada G, Claro JC et al. Oral direct Factor Xa inhibitors versus low-molecular-weight heparin to prevent venous thromboembolism in patients undergoing total hip or knee replacement: a systematic review and meta-analysis. Ann Intern Med 2012; 156: $710-719$
[19] Levy JH, Spyropoulos AC, Samama CM et al. Direct oral anticoagulants: new drugs and new concepts. JACC Cardiovasc Interv 2014; 7 : $1333-1351$

[20] Chatterjee P, Weitz Jl. Assessment and management of gastrointestinal hemorrhage in the setting of direct oral anticoagulants: the hematology perspective. Am J Gastroenterol Suppl 2016; 3: 29-35

[21] Veitch AM, Vanbiervliet G, Gershlick AH et al. Endoscopy in patients on antiplatelet or anticoagulant therapy, including direct oral anticoagulants: British Society of Gastroenterology (BSG) and European Society of Gastrointestinal Endoscopy (ESGE) guidelines. Endoscopy 2016; 48: $1-18$

[22] Pisters R, Lane DA, Nieuwlaat R et al. A novel user-friendly score (HASBLED) to assess 1-year risk of major bleeding in patients with atrial fibrillation: the Euro Heart Survey. Chest 2010; 138: 1093-1100

[23] Gage BF, Waterman AD, Shannon W et al. Validation of clinical classification schemes for predicting stroke: results from the National Registry of Atrial Fibrillation. JAMA 2001; 285: 2864-2870

[24] Pamukcu B, Lip GY, Lane DA. Simplifying stroke risk stratification in atrial fibrillation patients: implications of the CHA2DS2-VASc risk stratification scores. Age Ageing 2010; 39: 533- 535

[25] Robins JM, Rotnitzky A, Zhao LP. Estimation of regression coefficients when some regressors are not always observed. J Am Stat Assoc 1994; 427: $846-866$

[26] Connolly SJ, Ezekowitz MD, Yusuf S et al. Dabigatran versus warfarin in patients with atrial fibrillation. NEJM 2009; 361: 1139-1151

[27] Holster IL, Valkhoff VE, Kuipers EJ et al. New oral anticoagulants increase risk for gastrointestinal bleeding: A systematic review and meta-analysis. Gastroenterology 2013; 145: 105-112.e115

[28] Yoshio T, Nishida T, Hayashi Y et al. Clinical problems with antithrombotic therapy for endoscopic submucosal dissection for gastric neoplasms. World J Gastrointest Endosc 2016; 8: 756 - 762

[29] Douketis JD, Spyropoulos AC, Kaatz S et al. Perioperative bridging anticoagulation in patients with atrial fibrillation. NEJM 2015; 373 : $823-833$

[30] Kato M, Uedo F, Hokimoto S et al. Guidelines for gastroenterological endoscopy in patients undergoing antithrombotic treatment. Supplement revision of the use of anticoagulant drugs including direct oral anticoagulant drugs (DOACs). Gastroenterol Endosc 2017; 59: 1547-1558 (in Japanese)

[31] Heublein V, Pannach S, Daschkow K et al. Gastrointestinal endoscopy in patients receiving novel direct oral anticoagulants: results from the prospective Dresden NOAC registry. J Gastroenterol 2018; 53: 236 246

[32] Goldstein JL, Larson LR, Yamashita BD et al. Low molecular weight heparin versus unfractionated heparin in the colonoscopy peri-procedure period: a cost modeling study. Am J Gastroenterol 2001; 96 : $2360-2366$

[33] Jaff MR. Chronically anticoagulated patients who need surgery: can low-molecular-weight heparins really be used to "bridge" patients instead of intravenous unfractionated heparin? Catheter Cardiovasc Interv 2009; 74 (Suppl. 01): S17-S21 
- Supplementary Table 1 Post-procedural gastrointestinal bleeding and heparin bridge in each type of DOAC.

\begin{tabular}{|l|c|l|l|}
\hline & $\mathbf{n}$ & $\begin{array}{l}\text { Post-procedural gastrointestinal bleeding, } \\
\mathbf{n}(\%)\end{array}$ & $\begin{array}{l}\text { Heparin bridge, } \\
\mathbf{n}(\%)\end{array}$ \\
\hline Dabigatran & & $2(11.1)$ & $9(50.0)$ \\
\hline Rivaloxaban & 18 & $2(22.2)$ & $4(44.4)$ \\
\hline Apixaban & 9 & $1(12.5)$ & $5(62.5)$ \\
\hline Edoxaban & 8 & $1(50.0)$ & $0(0)$ \\
\hline DOAC, direct oralanticoagulant. & 2 & & \\
\hline
\end{tabular}

- Supplementary Table 2 Bleeding period after endoscopic procedures and the rate of recurrent bleeding.

\begin{tabular}{|c|c|c|c|}
\hline & VKAs & DOACs & $P$ value \\
\hline Bleeding period after endoscopic procedures (days), median (range) & $3.5(1-18)$ & $5(1-13)$ & 0.592 \\
\hline Recurrent bleeding/total bleeding, $\mathrm{n}(\%)$ & $3 / 26(11.5)$ & $0 / 6(0)$ & 0.382 \\
\hline
\end{tabular}

- Supplementary Table 3 Rate of post-procedural bleeding in patients taking VKAs or DOACs with and without heparin bridge therapy.

\begin{tabular}{|c|c|c|c|}
\hline & VKAs & DOACs & $P$ value* \\
\hline Without heparin bridge, $\mathrm{n}(\%)$ & $1 / 29(3.4)$ & $3 / 20(15.0)$ & 0.291 \\
\hline With heparin bridge, $\mathrm{n}(\%)$ & $25 / 129(19.3)$ & $3 / 17(17.6)$ & 1.000 \\
\hline
\end{tabular}

